\title{
BAT Locus
}

National Cancer Institute

\section{Source}

National Cancer Institute. BAT Locus. NCI Thesaurus. Code C13391.

Microsatellite repeats that have been used as markers of microsatellite instability. BAT 26, a poly(A) tract in MSH2 intron 5, is one example of a BAT locus. Presence of alterations in the BAT 26 locus, detected in stool samples, may be an easy and noninvasive detection method for colorectal cancer. 